\title{
Mortalidade materna de mulheres negras no Brasil
}

\author{
Maternal mortality among black \\ women in Brazil
}

\section{Abstract}

Every minute a woman dies in the world due to labor or complications of pregnancy. Maternal mortality is a public health problem in Brazil and affects the country's various regions unequally. Researchers agree that maternal death occurs mainly in women with lower income and less schooling. The racial issue emerges in the midst of socioeconomic issues. The analysis is hampered by the difficulty in understanding Brazil's official classification of race/color, which often impedes recording this information. Various Maternal Mortality Committees are applying the color item and reviewing their data. The current article analyzes various Maternal Mortality Committee reports, showing that the risk of maternal mortality is greater among black women (which encompasses two census categories, negra, or black, and parda, or brown), thus representing a major expression of social inequality. The article concludes with a review of political and technical recommendations to decrease maternal mortality.

Maternal Mortality; Race; Social Inequity

\section{Introdução}

A cada minuto morre no mundo uma mulher em virtude de complicações da gravidez e do parto. Ao fim de um ano, todas essas mortes somam 529 mil, a maioria das quais ocorre em países em desenvolvimento e poderia ser evitada. Para cada mulher que morre, outras trinta sofrem seqüelas ou problemas crônicos de saúde 1 .

No Brasil, a morte materna configura-se como um problema de saúde pública. Segundo o Ministério da Saúde (MS), as altas taxas de mortalidade materna compõem um quadro de violação dos direitos humanos de mulheres e de crianças, atingindo desigualmente aquelas das classes sociais com menor ingresso e acesso aos bens sociais, nas várias regiões brasileiras 2 .

O estudo da mortalidade materna tem desafios que superam análises individuais de cada caso e remetem a uma dimensão maior que é a população e suas questões de vida, saúde e doença, em uma sociedade onde não existe consenso sobre a igualdade de gênero e direitos reprodutivos. Tema essencial da saúde reprodutiva, o desafio está em relacioná-lo com a situação econômica e social e sua influência no desenvolvimento da humanidade, em um contexto explicito ou não, no qual as políticas populacionais nos colocam frente a frente com realidades diversas: fome e miséria no terceiro mundo, clonagem de seres humanos e novas 
tecnologias reprodutivas conceptivas e contraceptivas, para servir a alguns poucos.

É consenso que a mulheres acometidas pela morte materna são as de menor renda e escolaridade. O Comitê Estadual de Prevenção da Mortalidade Materna do Paraná (CEPMM-PR) 3 referiu, em 1997, que 52,5\% dos óbitos maternos ocorreram em mulheres com renda de 1 a 4 salários mínimos, $86,8 \%$ em mulheres com escolaridade de 1 a 4 anos e maior risco no grupo entre 35 a 39 anos. Outros estudos confirmam que as mulheres mais pobres também apresentam em geral maior número de filhos 4 e menor acesso à assistência 5 .

Juntamente com as questões sócio-econômicas emerge a questão racial. A análise é difícil de ser realizada por causa da dificuldade de entendimento da classificação raça/cor, já que existem várias tendências: por cor, por ascendência, por estratos sociais, havendo até quem acredite que não deva existir classificação. A maioria dos pesquisadores simplesmente se recusa a levar raça em conta quando são consideradas as causas da pobreza e da falta de oportunidades 6 .

A falta de entendimento das diferenças e diferenciais raciais/étnicos, da opressão de gênero e do racismo na manutenção, recuperação e perda da saúde em uma sociedade classista provoca "espanto" quando se menciona a saúde da mulher negra 7 . A despeito dos diferentes referenciais, os dados evidenciam que as mulheres negras vivem em piores condições de vida e saúde. Vários autores 8,9 afirmam que as mulheres negras têm os piores níveis de renda e escolaridade no Brasil.

Há inúmeros trabalhos sobre assistência ao pré-natal, parto e puerpério, porém só nos últimos anos vêm aumentando significativamente as pesquisas sobre raça/cor. A principal dificuldade consiste em considerar as diferentes variáveis relacionadas à assistência à saúde e condições de saúde da mulher e à condição social entre os diferentes grupos sociais e raciais ao se analisar a mortalidade materna.

O presente artigo almeja apresentar uma revisão não extensiva de estudos que relacionam os coeficientes de mortalidade materna por raça/cor no Brasil.

\section{Mortalidade materna no Brasil}

Reduzir a mortalidade materna no Brasil foi eleita como uma das metas do Projeto Objetivos de Desenvolvimento do Milênio, adotado pelo país em 2000 10. Já em 1984, reconhecendo a magnitude da tragédia da morte materna, o IV Encontro Internacional Mulher e Saúde definiu o 28 de maio como Dia Internacional de Luta contra a Morte Materna/Ação pela Saúde da Mulher. No Brasil, o MS referendou este dia, mediante portaria, como Dia Nacional de Redução da Mortalidade Materna, data em que devem ser realizadas avaliações dos programas com tal finalidade, reconhecendo a necessidade de ações amplas para atingir esse objetivo. Dentre essas ações, destaca-se a criação do Comitê Nacional de Combate à Morte Materna, cujo papel essencial é o estudo da mortalidade materna, que servirá de base para a construção do coeficiente ou taxa de mortalidade materna. Além da investigação para fins epidemiológicos, há também o papel estratégico de monitorar a implantação de políticas públicas para a prevenção e reparação de casos de morte materna. Os comitês, nesse aspecto, devem agir em parceria e consonância com outros setores públicos para a responsabilização institucional 11 .

O conhecimento do coeficiente ou razão de mortalidade materna é indispensável para o planejamento e gerência das ações de saúde, em qualquer instância, sendo obtido por meio da análise das declarações de óbito e, se possível, mediante investigações da causa básica do óbito de mulheres em idade fértil. Os resultados do estudo desse coeficiente são de alta abrangência, levam em conta aspectos bastante diversos que envolvem a morte materna, fornecendo dados específicos da assistência pré-natal, ao parto e ao puerpério, sem deixar de lado questões como a gravidez indesejada, aborto entre outros. Atrash et al. 12, em levantamento de mortalidade materna de 14 países, referem que devem ser usadas múltiplas fontes de informação (comportamento individual, acesso aos serviços, entre outras), para entender a contribuição de fatores de risco, e as estratégias de intervenção devem ser avaliadas continuamente para assegurar sua efetividade.

Villela 13 cita que, no Brasil, há grande disponibilidade de dados, com diversas possibilidades de desagregação. No entanto, a qualidade dos dados nem sempre é boa, nem todos os eventos que devem ser monitorados para verificar a efetividade das ações de saúde das mulheres são passíveis de acompanhamento, em face dos dados disponíveis. Nesse sentido, no que se refere à mortalidade materna, não há diferença, pois qualquer análise requer informações de duas bases de dados: Sistema de Informações sobre Mortalidade (SIM) e Sistema de Informações sobre Nascidos Vivos (SINASC), e esbarra em questões desde o preenchimento das Declarações de Óbito (DO) e Declarações de Nascido Vivo (DNV) pelos profissionais de 
saúde, à implantação/digitação dos respectivos programas.

Considerando apenas os óbitos declarados, o MS cita que, de 1996 a 1998, houve um acréscimo da razão de mortalidade materna, em especial pelo aumento por causas obstétricas indiretas. Sugere-se, assim, que esse crescimento deveu-se a uma melhoria na qualidade das informações, provavelmente associada ao processo de investigação de óbitos de mulheres em idade fértil, por parte dos comitês de morte materna. De 1999 a 2001, essa razão apresentou queda. Neste período, a questão da mortalidade materna ganhou maior visibilidade e vários processos estaduais e municipais foram deflagrados para este enfrentamento 2 .

Diversos estudos estimaram a mortalidade materna no Brasil considerando a subnotificação e encontraram fator de correção até 2 , ou seja, para cada óbito materno somar um não declarado. Volochko 14 encontrou razão de mortalidade materna, para o país, de 66,08/100 mil nascidos vivos, em 1980, e 64,85 em 1998. Laurenti et al. 15 calcularam fator de correção de 1,67 para a mortalidade materna, que é utilizado oficialmente hoje pelo MS, tendo-se para 2001 e 2002 taxa de mortalidade materna de 69,19 e 73,05/ 100 mil nascidos vivos respectivamente, com 1.528 e 1.603 óbitos maternos (Indicadores e dados básicos para a saúde. http: //www.datasus.gov.br, acessado 08/Ago/2005).

Os dados oficiais 2 evidenciam que as doenças hipertensivas, seguidas das síndromes hemorrágicas, são as principais causas de mortalidade materna no país. Ambas mantêm-se, há décadas, como as duas principais causas, ora seguidas pelas infecções puerperais e o aborto, ora pelas doenças do aparelho cardiovascular, complicadas pela gravidez, parto ou puerpério.

Mello-Jorge 16 refere óbitos maternos dos 14 aos 45 anos, chegando à média de idade de 28,6 anos. No grupo estudado, 3,6\% mulheres eram analfabetas; $25 \%$ eram casadas, $32 \%$ viviam em união consensual e $41 \%$ não tinham companheiro, sendo a maioria "do lar".

A mortalidade materna difere segundo classe social, níveis de renda, escolaridade, entre outros. Portanto, a análise desse indicador é qualitativamente melhor quando algumas variáveis subjacentes às condições sócio-econômicas da população são explicitadas, tais como renda, idade, escolaridade e raça.

\section{Mortalidade materna das mulheres negras}

O Centers for Diease Control and Prevention 17 reporta a diminuição dos coeficientes de morte materna nos Estados Unidos de 319,8 para $5,7 / 100$ mil nascidos vivos entre as mulheres brancas e de 781,7 para 18,6/100 mil nascidos vivos entre as mulheres negras, no período de 1940 a 1990. Essa razão 2 a 4 vezes maior para mulheres negras explica-se pelo grande número de gravidez com morbidade, dificuldade de acesso aos serviços de saúde e uso destes, além da qualidade da assistência prestada, ou cuidados recebidos.

Atrash et al. 12 afirmam que ser membro de uma minoria étnica em si mesmo não explica a razão para essa disparidade; porém, é importante ter tal informação quando fazemos a prevenção. Em revisão de literatura os autores encontraram risco de mortalidade materna variando de 3,7 para não germânicas na Bavária, 4,3 para negras no Estados Unidos, 10,0 para aborígines na Austrália, entre outros.

Na complexidade que é o estudo da mortalidade materna, que permite avaliar desde as questões sociais até o acesso aos serviços de saúde, a variável raça/cor deve ser bem analisada, principalmente porque os óbitos maternos ocorrem, em sua maioria, em mulheres de classes sociais mais baixas, com baixa escolaridade e baixos salários.

No Brasil, segundo o Instituto Brasileiro de Geografia e Estatística (IBGE), os estudos devem classificar o quesito cor em amarela, branca, parda, preta e indígena. No primeiro trabalho nacional conhecido com este recorte e óbitos maternos ocorridos em 1997 no Rio Grande do Norte, Mato Grosso e Pará, Tanaka \& Mitsuiki 19 citam que prevaleceu a cor branca, com $28,5 \%$ dos óbitos, porém, ao se considerarem as cores pardas, morenas e morenas claras como sendo uma só, a soma destas atingiu 51,5\% dos óbitos. No Estado do Pará, excluindo-se as mortes maternas sem esta informação, todas foram consideradas pardas (morena e morena clara $62,5 \%)$.

Tendo em vista a distribuição demográfica no Brasil, sendo os Estados do Sul conhecidos pela colonização principalmente européia, Martins 19 refere que, no Estado do Paraná, no período de 1993 a 1998, a maioria dos óbitos ocorreu entre as mulheres de cor branca $(53,4 \%)$. As negras, somatório das pardas e pretas, totalizaram $17,9 \%$, e a raça amarela, 1,4\%. Entretanto, utilizando o Censo do IBGE realizado em 1991 20, ao comparar a distribuição da população feminina e o número de mortes maternas por cor, 
para 1993, observou-se que o risco relativo de morte destas mulheres foi 7,4 vezes maior nas pretas e 5 vezes maior entre as amarelas, quando comparadas com as brancas. Ainda neste estudo, na população de mulheres negras, apenas $2,2 \%$ eram pretas, porém $8,2 \%$ dos óbitos maternos foram destas. As mulheres de raça/cor amarela apresentaram características sócioeconômicas e demográficas melhores do que as brancas; as pretas, as piores. No entanto, o risco de morte de ambas foi muito elevado, sugerindo que deve existir relação entre raça, doença, condições de vida e mortalidade materna e que se faz necessário maior aprofundamento no tema.

Martins 21, utilizando os dados de óbitos maternos ocorridos nas capitais brasileiras, no Município de São Paulo, em três municípios da Bahia (Alagoinhas, Feira de Santana e Salvador) e no Estado do Paraná, presentes no "Estudo da Mortalidade de Mulheres de 10 a 49 Anos com Ênfase na Mortalidade Materna", de Laurenti et al. 15, elaborou o estudo Diferenciais Raciais nos Perfis e Indicadores de Mortalidade Materna para o Brasil.

Como contraponto aos dados do Paraná 22, onde, segundo Censo do IBGE de 2000 23, a população negra corresponde a $21,1 \%$, sendo $18,2 \%$ pardos e 2,8\% pretos, algumas cidades da Bahia, como Alagoinhas, Feira de Santana e Salvador, foram escolhidas para o estudo de Martins 21 , por o Estado contar, de acordo com a mesma fonte, com $73,2 \%$ de população negra (pardos 60,1\% e pretos 13\%). Observou-se que, mesmo com elevado percentual de nascidos vivos ignorados, atingindo $25,7 \%$, a razão de mortalidade materna para as mulheres pretas nestes municípios é sempre maior. Calculando-se o risco relativo em relação às negras, maior população, estas ficaram com 3,7, seguidas das mulheres brancas, com 2,6. Excluindo-se os óbitos tardios, o autor encontrou, para as capitais, que as mulheres brancas apresentam a menor razão e mortalidade, 48,73/100 mil nascidos vivos, seguidas das negras, com 72,61, sendo 65,07 para as pardas e 227,60 para as pretas.

A razão de mortalidade materna é maior na Região Nordeste, com 71,32/100 mil nascidos vivos, e menor na região sul com 41,70/100 mil nascidos vivos. Traduzindo a razão em risco de morte materna, as mulheres negras, as pardas e as pretas, em relação às brancas, apresentaram, respectivamente, risco 1,7 e 1,8; 1,5 e 1,6; 5,5 a 7,4 vezes maior de óbito.

Estes resultados concordam com outros encontrados por Chor \& Lima 24. Estas citam que cabe destacar ainda a importante diferença entre as taxas de mortalidade materna nas capi- tais, cujas informações são consideradas de melhor qualidade do que aquelas relativas ao conjunto do país. Entre as mulheres pretas, em 2001, a taxa foi cerca de sete vezes maior (275/100 mil nascidos vivos) do que entre mulheres brancas (43/100 mil nascidos vivos), ou pardas (46/ 100 mil nascidos vivos).

Em estudo sobre a mortalidade de 1999 para o Estado de São Paulo, Batista 25 identificou que a mortalidade materna é a segunda causa de morte entre as mulheres pretas. Ao analisar a totalidade dos óbitos (349) destas, constatou que supera a das brancas em 5,6 vezes, com coeficiente de 212,80/ 100 mil nascidos vivos. A razão de mortalidade materna para o Estado foi $47,81 / 100$ mil nascidos vivos no ano analisado.

Segundo o Relatório do Comitê de Morte Materna da Prefeitura Municipal de São Paulo, no ano 200026 , não foi possível determinar a raça/cor da falecida em cinco $(4,4 \%)$ casos. A cor branca foi a mais freqüente (61 casos - 53,9\%), seguida da parda (32 casos $-28,3 \%$ ), da preta ( 14 casos $-12,4 \%)$ e $1(0,9 \%)$ caso da raça amarela. Em 1999 os percentuais foram muito próximos, e as ignoradas totalizaram sete casos $(5,5 \%)$.

Analisando a base de dados do Comitê de São Paulo, Martins 21 encontrou maior razão de mortalidade materna para as mulheres pretas, atingindo 562,35/100 mil nascidos vivos, com risco relativo de 6,7 . A razão total foi 56,15/100 mil nascidos vivos.

É elevado o percentual de nascidos vivos de raça ignorada no município no período estudado, atingindo $45,73 \%$, o que compromete avaliação desse resultado. Entretanto, as mulheres pretas apresentam maior razão de mortalidade materna em São Paulo, também na base capitais, respectivamente 301,20 e 323,62/100 mil nascidos vivos das Secretarias Municipais de Saúde ou MS.

\section{O quesito cor}

Assim como no Município de São Paulo, no Estado do Paraná 20 o quesito cor dos óbitos maternos é investigado desde 1993, constituindo-se em importante fonte de informação para o estudo da mortalidade materna de mulheres negras.

Em 2002, o Comitê de Morte Materna do Estado do Paraná revisou as fichas de investigação dos óbitos maternos, preenchendo as variáveis em branco, especialmente idade, raça e escolaridade. O percentual de óbitos maternos de raça ignorada no período de 1993 a 1997 correspondia a $27,4 \%$ e baixou para $9,2 \%$. Ao mesmo tempo, a Secretaria de Estado da Saúde 
desenvolveu um trabalho com as regionais de saúde para melhorar o preenchimento da DNV, com resultados positivos; apenas $2,5 \%$ dos nascidos vivos são de raça ignorada. Com essa melhoria dos dados, observa-se, na Tabela 1, que aumentou de 7,4 19 para 8,2 o risco de óbito materno das mulheres pretas em relação às brancas no Estado do Paraná 21.

Outro comitê que investiga e analisa óbitos maternos e raça é o do Estado do Rio de Janeiro. O relatório do Comitê de Morte Materna da Secretaria Estadual de Saúde do Rio de Janeiro (SES-RJ) 27 cita que a taxa em mulheres negras por causa considerada materna foi 1,8 vez maior que em mulheres brancas em 1999.

À medida que há melhoria da informação, a tendência é o aumento da confirmação estatística dos riscos para as minorias étnicas; foi o que ocorreu com os dados do Paraná e certamente com os do Rio de Janeiro. Para 2000, a SES-RJ refere que a taxa de mortalidade materna em mulheres negras foi 2,3 vezes maior do que a taxa das mulheres brancas (39/100 mil nascidos vivos para brancas e 100/100 mil nascidos vivos para negras) 27 .

Independente da base de dados, do percentual de nascidos vivos com cor ignorada e do período do estudo, as pretas sempre apresentam a maior razão de mortalidade materna. Sendo assim, este estudo conclui que existe grande diferencial para a mortalidade materna das mulheres negras, dentre as quais as pretas apresentaram:

a) O maior percentual de correção, $44,4 \%$; de óbitos tardios, $64,7 \%$; de solteiras, $61,19 \%$; de renda de um a dois salários mínimos, 60,5\%; b) As maiores razões de mortalidade materna: 562,35/100 mil nascidos vivos em São Paulo, 197,77/100 mil nascidos vivos na Bahia, 407,05/ 100 mil nascidos vivos no Paraná e 227,60/100 mil nascidos vivos na base capitais, excluindose os óbitos tardios;

c) Risco relativo de morte materna variando de 3,7 (Bahia) a 8,2 (Paraná);

d) Risco relativo de morte materna por doença hipertensiva específica da gravidez de 8,2 , com razão de mortalidade materna de 85,77/ 100 mil nascidos vivos, tendo as brancas apresentado 10,36 e o total sendo 11,37/100 mil nascidos vivos;

e) Risco relativo de morte materna por hipertensão arterial sistêmica de 18,2, com razão de mortalidade materna de 50,03/100 mil nascidos vivos, tendo as brancas apresentado 2,75 e o total sendo 3,53/100 mil nascidos vivos.

\section{Principais recomendações para redução da mortalidade materna de mulheres negras no Brasil}

Apesar dos avanços no país em relação à redução da mortalidade materna, muito ainda precisa ser feito para que as brasileiras tenham o direito à saúde plenamente assegurada, especialmente as mulheres negras.

Com o advento do Pacto Nacional de Redução da Mortalidade Materna e Neonatal 2, certamente a mortalidade materna ganha visibilidade e conseqüentemente espera-se que as questões relativas a esse tema sejam discutidas e implementadas, como, por exemplo:

Distribuição de óbitos maternos, nascidos vivos, razão e risco de morte materna segundo raça. Paraná, Brasil, 2000 a 2002.

\begin{tabular}{|c|c|c|c|c|c|c|c|}
\hline \multirow[t]{2}{*}{ Raça } & \multicolumn{2}{|c|}{ Óbitos maternos } & \multirow[t]{2}{*}{ Óbitos tardios } & \multicolumn{2}{|c|}{ Nascidos vivos } & \multirow{2}{*}{$\begin{array}{c}\text { Razão de mortalidade } \\
\text { materna (por } 100.000 \\
\text { nascidos vivos) }\end{array}$} & \multirow[t]{2}{*}{ Risco relativo } \\
\hline & $\mathrm{n}$ & $\%$ & & $\mathrm{n}$ & $\%$ & & \\
\hline Amarela & 1 & 0,3 & - & 1.145 & 0,2 & 87,33 & 1,7 \\
\hline Branca & 250 & 69,4 & 19 & 468.997 & 91,8 & 49,25 & 1,1 \\
\hline Negra & 76 & 21,1 & 10 & 26.894 & 5,3 & 245,4 & 4,9 \\
\hline Parda & 54 & 15,0 & 6 & 22.472 & 4,4 & 213,59 & 4,3 \\
\hline Preta & 22 & 6,1 & 4 & 4.422 & 0,9 & 407,05 & 8,2 \\
\hline Indígena & - & - & - & 1.175 & 0,2 & - & - \\
\hline Ignorada & 33 & 9,2 & 8 & 12.751 & 2,5 & 196,06 & - \\
\hline Total & 360 & 100,0 & 37 & 510.962 & 100,0 & 63,21 & - \\
\hline
\end{tabular}

Fonte: Comitê Estadual de Prevenção da Mortalidade Materna do Paraná.

* Excluídos os óbitos tardios. 
a) Atuação das secretarias estaduais e municipais, seguindo a Política Nacional de Atenção à Saúde da Mulher, incluindo a capacitação dos gestores;

b) Políticas públicas e financiamento do setor saúde, dentre outros, implantando as Portarias Ministeriais que direcionam a assistência e trazem financiamento, como o Programa de $\mathrm{Hu}$ manização ao Pré-natal e Nascimento;

c) Atenção ao aborto: monitoramento dos trâmites do projeto de Lei da Comissão Tripartite na Câmara Federal;

d) Ampliação e consolidação do controle social; e) Qualidade da assistência prestada pelos profissionais de saúde. É necessária formação profissional humanizada, educação continuada e permanente;

f) Utilização de estratégias dos direitos humanos, como o inquérito civil público e termo ou compromisso de ajustamento de conduta, procedimentos administrativos iniciados e supervisionados pelo Ministério Público; a ação civil pública, procedimento judicial que também po- de ser iniciado pelo Ministério Público, organizações não governamentais e outros órgãos; a ação civil indenizatória, de caráter individual, que pode ser apresentada pelos familiares;

g) Apoio a projetos como o Qualificando o Controle Social para a Redução da Mortalidade Materna e criação da Associação Nacional Familiares das Vítimas de Morte Materna, desenvolvidos pela Rede Feminista de Saúde; apoio a grupos de teatro como Loucas de Pedra Lilás e outras organizações de mulheres atuando desde o treinamento de parteiras - como o Curumim, CAIS do Parto e Rede Nacional de Parteiras Tradicionais - até o desenvolvimento de atividades de monitoramento da Plataforma de Ação do Cairo.

Para as mulheres negras, a existência do quesito cor nos documentos oficiais foi o primeiro passo para redução das desigualdades, entretanto ainda são necessários o treinamento e a sensibilização dos profissionais de saúde sobre a importância da atenção à saúde, registro e análise dos dados sobre raça/cor/etnia.

\section{Resumo}

A cada minuto uma mulher morre no mundo em decorrência do trabalho de parto ou complicações da gravidez. A mortalidade materna configura-se no Brasil como um problema de saúde pública, atingindo desigualmente as várias regiões brasileiras. É consenso que a mulheres acometidas pela morte materna são as de menor renda e escolaridade. Juntamente com as questões sócio-econômicas, emerge a questão racial. A análise é difícil de ser realizada em virtude da dificuldade de entendimento da classificação raça/cor que muitas vezes impede o registro dessa informação. Vários Comitês de Morte Materna estão utilizando o quesito cor e revisando seus dados. Este artigo analisa vários relatórios de Comitês de Morte Materna, mostrando que o risco de mortalidade materna é maior entre as mulheres negras, o que inclui as pretas e pardas, configurando-se em importante expressão de desigualdade social. Ao final, apresenta-se uma revisão de recomendações para diminuição da Mortalidade Materna, enfatizando ações políticas e técnicas que possam contribuir para tal. 


\section{Referências}

1. United Nations Population Fund. Maternal mortality update 2004: delivering into good hands. http:/ / www.unfpa.org (acessado em 26/Fev/2006).

2. Ministério da Saúde. Pacto nacional pela redução da mortalidade materna e neonatal. Brasília: Ministério da Saúde; 2004.

3. Comitê Estadual de Prevenção da Mortalidade Materna. Relatório trienal 1994-96. Mortalidade materna no Paraná, do anonimato à ação. Curitiba: Comitê Estadual de Prevenção da Mortalidade Materna; 1997.

4. Berquó E. Aspectos demográficos da população feminina. In: Seminário Nacional: Gestão em Saúde da Mulher. Brasília: Secretaria de Atenção a Saúde, Ministério da Saúde; 2004. p. 8-13.

5. Perpétuo IHO. Raça e acesso às ações prioritárias na agenda da saúde reprodutiva. Jornal da Rede Saúde 2000; (22):24-8.

6. Heringer R. Desigualdades raciais no Brasil: síntese de indicadores e desafios no campo das políticas públicas. Cad Saúde Pública 2002; 18 Suppl: 57-65.

7. Oliveira F. Saúde da população negra: Brasil ano 2001. Brasília: Organização Pan-Americana da Saúde; 2003.

8. Secretaria de Políticas de Saúde. Manual de doenças mais importantes, por razões étnicas, na população brasileira afro-descendente. Brasília: Ministério da Saúde; 2001.

9. Sant'Anna W. Dossiê assimetrias raciais no Brasil. Belo Horizonte: Rede Nacional Feminista de Saúde, Direitos Sexuais e Direitos Reprodutivos; 2003.

10. Objetivos de Desenvolvimento do Milênio. Relatório Nacional de Acompanhamento. http:// www.planalto.gov.br (acessado em 21/Mar/2006).

11. Galli B. Panorama da mortalidade materna no Brasil. In: Cidadania, Estudo, Pesquisa, Informação e Ação, organizador. Promovendo a maternidade segura através dos direitos humanos. Rio de Janeiro: Cidadania, Estudo, Pesquisa, Informação e Ação/Organização Mundial da Saúde; 2003. p. 97-109.

12. Atrash HK, Alexander AS, Berg CJ. Maternal mortality in developed countries: Not just a concern of the past. Obstet Gynecol 1995; 86 (4 Part 2): 700-5.

13. Villela WO. Monitoramento da plataforma de ação da Conferência Internacional sobre População e Desenvolvimento no Brasil. Jornal da Rede Saúde 2004; (26):1-12.

14. Volochko A. A mensuração da mortalidade materna no Brasil. In: Berquó E, organizador. Sexo \& vida: panorama da saúde reprodutiva no Brasil. Campinas: Universidade Estadual de Campinas; 2003. p. $229-56$
15. Laurenti R, Mello-Jorge MHP, Gotlieb SLD. Estudo da mortalidade de mulheres de 10 a 49 anos, com ênfase na mortalidade materna. Brasília: Ministério da Saúde/Organização Pan-Americana da Saúde/Universidade de São Paulo; 2002.

16. Mello-Jorge MHP. Mortalidade da mulher adulta no Brasil. In: Seminário Nacional: gestão em saúde da mulher. Brasília: Secretaria de Atenção à Saúde, Ministério da Saúde; 2004. p. 14-26.

17. Centers for Disease Control and Prevention. Differences in maternal mortality among black and white women - United States, 1990. MMWR Morb Mortal Wkly Rep 1995; 44:6-7,13-14.

18. Tanaka ACd'A, Mitsuiki L. Estudo da magnitude da mortalidade materna em 15 cidades brasileiras. São Paulo, 1999. http://www.saude.gov.br/ programas/mulher/ (acessado em 10/Out/2003).

19. Martins AL. Mulheres negras e mortalidade materna no Estado do Paraná, entre 1993 a 1998 [Dissertação de Mestrado]. Ponta Grossa: Universidade Estadual de Ponta Grossa; 2000.

20. Instituto Brasileiro de Geografia e Estatística. Censo demográfico 1991 - características gerais da população e instrução. Rio de Janeiro: Instituto Brasileiro de Geografia e Estatística; 1992.

21. Martins AL. Diferenciais raciais nos perfis e indicadores de mortalidade materna para o Brasil. In: XIV Encontro da Associção Brasileira de Estudos Populacionais. Caxambu: Associação Brasileira de Estudos Populacionais; 2004.

22. Comitê Estadual de Morte Materna do Paraná. Relatório do triênio 2000-2002. Curitiba: Comitê Estadual de Morte Materna; 2004.

23. Instituto Brasileiro de Geografia e Estatística. Censo demográfico 2000 - características gerais da população. Rio de Janeiro: Instituto Brasileiro de Geografia e Estatística; 2001.

24. Chor D, Lima CRA. Aspectos epidemiológicos das desigualdades raciais em saúde no Brasil. Cad Saúde Pública 2005; 21:1586-94.

25. Batista LE. Mulheres e homens negros: saúde, doença e morte [Tese de Doutorado]. Araraquara: Universidade Estadual Paulista Júlio de Mesquita Filho; 2002.

26. Prefeitura Municipal de São Paulo. Relatório do Comitê de Morte Materna de 2000. http: / / www. prefeitura.saude.sp.gov.br/programas/mulher (acessado em 10/Ago/2005).

27. Secretaria de Estado da Saúde do Rio de Janeiro. Relatório do comitê de morte materna de 2000. Rio de Janeiro: Secretaria de Estado da Saúde; 2002.

Recebido em 04/Mai/2006 Aprovado em 27/Jul/2006 